\title{
The Impact of Microplastics on Salp Feeding in the Tropical Pacific
}

\author{
Wing Y. Chan and Jan Witting
}

\section{Abstract}

While phytoplankton remove atmospheric $\mathrm{CO}_{2}$ through photosynthesis, salp (filter-feeding pelagic tunicates) feed on them and transport this newly-fixed carbon to the deep ocean via their fast-sinking fecal pellets. For this reason, the feeding activities of salp are important to the carbon cycle. However, the discovery of the Great Pacific Garbage Patch has raised concerns regarding the effects of plastics on marine organisms. Specifically, although the impact of macroplastics on marine mammals has been well-documented, the impact of microplastics on filter-feeders has yet to be investigated.

This marks the first study to examine the impact of microplastics on salp feeding using fluorescent polyethylene microspheres in a series of feeding experiments under controlled conditions. Results indicate that larger-sized salp (i.e. $\geq 3 \mathrm{~cm}$ ) are vulnerable to microplastics ingestion at a high microplastic concentration (i.e. 4.2 microspheres/ ml), while smaller-sized salp (i.e. $<3 \mathrm{~cm}$ ) are more vulnerable to the clogging of their feeding apparatus at medium to high microplastic concentrations (i.e. 3.2 and 4.2 microspheres/ $\mathrm{ml}$ ).

Keywords: Salp, Feeding, Microplastics, Fluorescent Polyethylene Microspheres

\section{Introduction}

Phytoplankton grazers (e.g. krill and salp) are important to the carbon cycle as they package and transfer the $\mathrm{CO}_{2}$ fixed by phytoplankton to the deep ocean via their fecal pellets. This process of downward carbon flux is crucial to the maintenance of a steady atmospheric $\mathrm{CO}_{2}$ level. Specifically, salp are one of the most efficient carbon packagers in the biological pump (Fortier et al., 1994). This is because their fecal pellets have a relatively higher carbon content and a faster sinking rate (i.e. $\sim 400-900 \mathrm{~m} /$ day) than other major grazers such as krill and pteropods (Ramaswamy et al., 2005). During a phytoplankton bloom in the Southern Ocean in 1994, salp were estimated to be able to export up to 
$88 \mathrm{mg}$ of carbon $/ \mathrm{m}^{2} /$ day to the deep ocean (Pakhomov \& Pakhomov, 1998). In addition, diel vertical migration of some salp species also helps to accelerate this downward carbon flux (Nishikawa \& Tsuda, 2001).

Since the discovery of the Great Pacific Garbage Patch (a 700,000 km² region of floating plastic debris in the North Pacific Ocean) by Capt. Charles Moore in 1997, the issue of marine plastics has received considerable scientific attention (Kaiser, 2010). Much research has been done since then on the impact of macroplastics on marine mammals, and $\sim 100,000$ marine mammals are estimated to die each year due to plastic ingestion or entanglement in the North Pacific Ocean (Moore et al., 2001). The effect of microplastics (i.e. plastics $<5 \mathrm{~mm}$ ) on filter-feeders such as salp, however, has not been investigated (Thompson et al., 2004). To date, only one study has documented the embedment of microplastics in the body of one salp (Moore et al., 2001). Since macroplastics disintegrate into microplastics over time, the amount of microplastics in the ocean is likely to increase in the future, as well as their negative impacts on pelagic filler-feeders (Doyle et al., 2010).

The design of the feeding mechanism of pelagic filter-feeders like salp renders these animals particularly vulnerable to marine microplastics for two reasons. Firstly, salp are unlikely to be able to distinguish between microplastics and their real prey. Salp feed by filtering particles through their mucous net - a net that is continuously secreted in their pharyngeal chamber (Harbison \& McAlister, 1979). When salp swim through the water column, they contract their circular muscle bands to allow water to enter their oral opening, and to pass through the meshes of their mucous net (Sutherland et al., 2010) (Figure 1) The mucous net then moves posteriorly toward their oesophagus, carrying food particles to their stomach (Sutherland et al., 2010). This feeding mechanism is not modulated in response to particle types, sizes and densities, and is therefore unlikely to allow the salp to distinguish between microplastics and phytoplankton (Harbison et al., 1986).

Secondly, the feeding apparatus of salp is susceptible to clogging resulting from the ingestion of oversized prey or the presence of high particle concentrations (Vargas \& Madin, 2004). Salp are unable to remove unwanted materials from their feeding apparatus and therefore the clogging of it often results in the death of the animal (Harbison \& McAlister, 1979). It has been reported that smallsized salp were unable to efficiently ingest large prey, such as large diatoms and ciliates (Vargas \& Madin, 2004). It has also been reported that phytoplankton blooms (which result in a high particle concentration in the ocean) are often associated with salp population collapse, possibly due to the clogging of their filtering apparatus (Pakhomov \& Pakhomov, 1998). Consequently, the presence of pelagic microplastics (especially at a high concentration) may have devastating impacts on salp populations. 


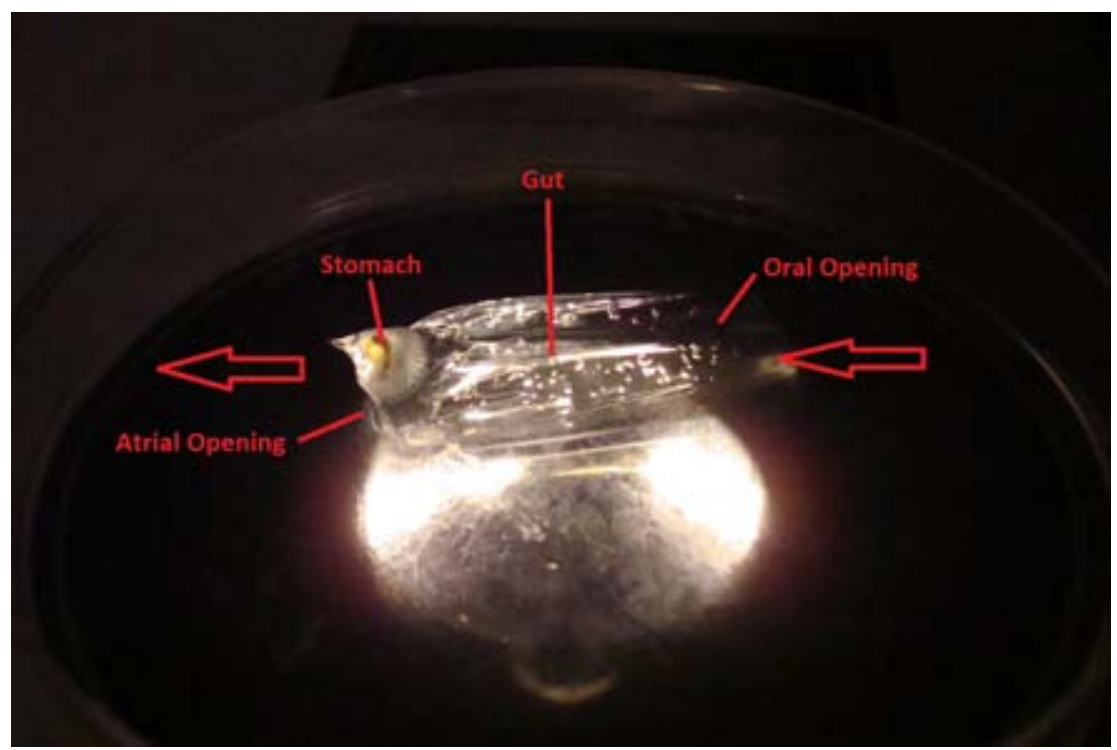

Figure 1. The feeding mechanism of salp. The arrow indicates the direction of water passing through their body.

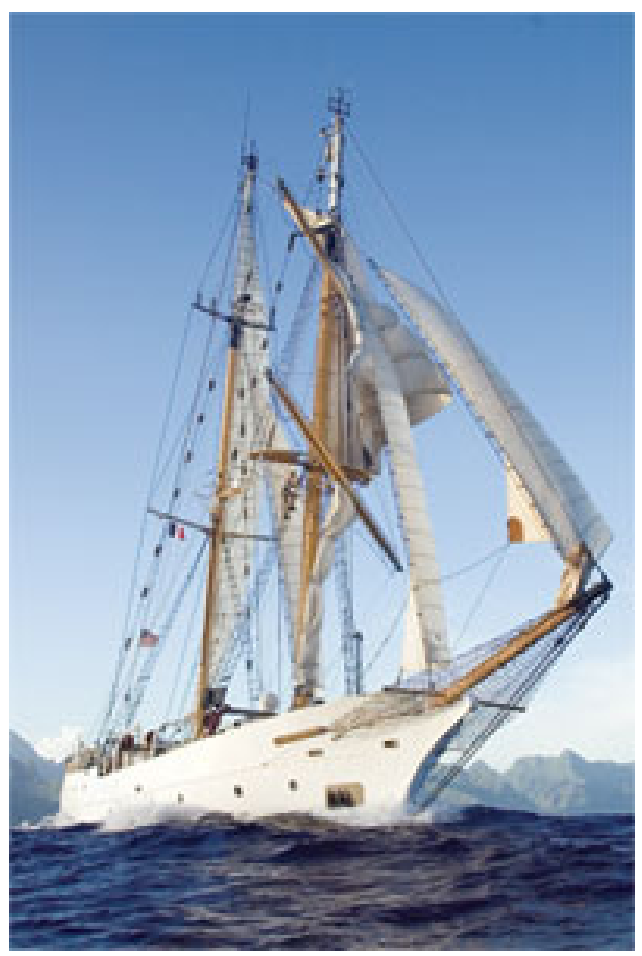

Figure 2. SSV Robert C. Seamans. 
This study was conducted as part of the Sea Education Association (USA) S-238 cruise on board the SSV Robert C. Seamans from Hawaii to Tahiti between 15 November and 23 December 2011 (Figure 2). While this study examined on the impact of microplastic salp feeding, 20 other studies were conducted simultaneously on board with different focuses (e.g. ocean circulation, ocean nutrient content, etc.) to achieve a broader understanding of the ocean's role in the global carbon cycle and climate change.

\section{Materials and methods}

\subsection{Salp collections}

Twelve live salp were collected at six stations during a cruise from Hawaii to Tahiti (Figure 3). Salp were collected using neuston and meter nets ( $333 \mu \mathrm{m}$ mesh size) deployed at night. The neuston nets were towed at the sea surface for about 30 minutes and the meter nets were towed at a depth of $300 \mathrm{~m}$ for about 20 minutes. In order to obtain healthy, normal behaving salp for the feeding experiments, salp species with a robust body were targeted for collection. In the first and second week of the cruise, the health status of different salp species collected were compared and three species were identified to be most suitable for the feeding experiment. These included Thalia longicauda, Ritteriella amboinensis and Cyclosalpa affinis. All salp used in the study were solo stage salp of the above three species and their size ranged from $1.4 \mathrm{~cm}$ to $5.1 \mathrm{~cm}$.

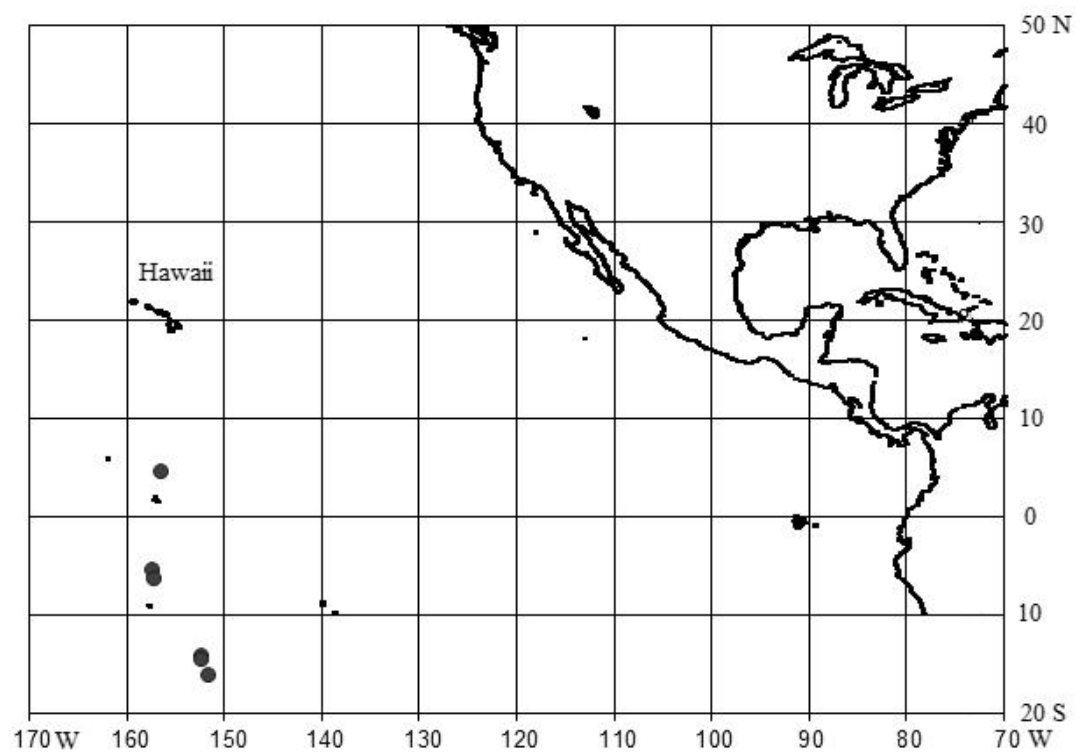

Figure 3. Study area in the tropical Pacific showing the locations of the 


\subsection{Feeding experiments}

Fluorescent polyethylene plastic microspheres (manufactured by Cospheric LLC) were used in this study to simulate the presence of suspended microplastics. These microspheres ranged from $90-106 \mu \mathrm{m}$ in size and were $98.27 \mu \mathrm{m}$ on average of 100 microspheres. A stock solution made of fluorescent microspheres and sea water was created in advance with a concentration of 200 microspheres/ $\mathrm{ml}$. Each slap was carefully separated from other tow net contents and transferred into a $950 \mathrm{ml}$ feeding jar. This jar was then placed into a larger-sized aquarium to help to minimize the extent of free surface effect on the animals. A selected amount of stock solution (10, 15 or $20 \mathrm{ml}$ ) was then added to the jar, giving a low, medium or high microsphere concentration (i.e. 2.1, 3.2 or 4.2 microspheres/ ml respectively). Different microsphere concentrations were used in this study to investigate how particle density may affect salp feeding.

Salp were allowed to feed for 1 hour and 15 minutes before they were removed from the jar to estimate the amount of microsphere ingested. The gut, stomach and oral opening of each salp were carefully examined under a dissection microscope (x 30 magnification) and the amount of microspheres ingested was manually counted and digitally recorded. Ingestion of microspheres was defined as the presence of microspheres in a salp's gut or stomach. The presence of microsphere in its oral opening was not defined as ingestion, rather, it was counted as a separate category. A total of six feeding experiments were conducted on 12 live salp.

\section{Results}

Ingestion of fluorescent microspheres occurred only in larger-sized salp(i.e. $\geq 3 \mathrm{~cm}$ ) that were subjected to a high microsphere concentration (i.e. 4.2 microspheres/ $\mathrm{ml}$ ) (Figure 4.1). These include Salp 7 (3 cm), Salp $11(4.2 \mathrm{~cm})$ and Salp 12 (3.5 $\mathrm{cm}$ ) (Figure 5.1 and 5.2). Salp 7, in particular, had packaged a large amount of microspheres into its fecal pellets. No microsphere ingestion was found in larger-sized salp that were subjected to a low microsphere concentration (i.e. 2.1 microspheres/ $\mathrm{ml}$ ) (Figure 4.1). On the other hand, ingestion of microspheres was not found in any smaller-sized salp (i.e. $<3 \mathrm{~cm}$ ) at all microsphere concentrations (Figure 4.1 and Figure 6). Clusters of microspheres, instead, were observed in the oral openings of all smaller-sized salp (except Salp 10, see remark \# on Table 1) (Figure 4.2 and Figure 7). Clusters of microspheres were also observed in the oral openings of some larger-sized salp, although this happened at a much lower frequency than for smaller-sized salp. The results of the feeding experiments are summarized in Table 1 and Figure 4.1 and 4.2. 


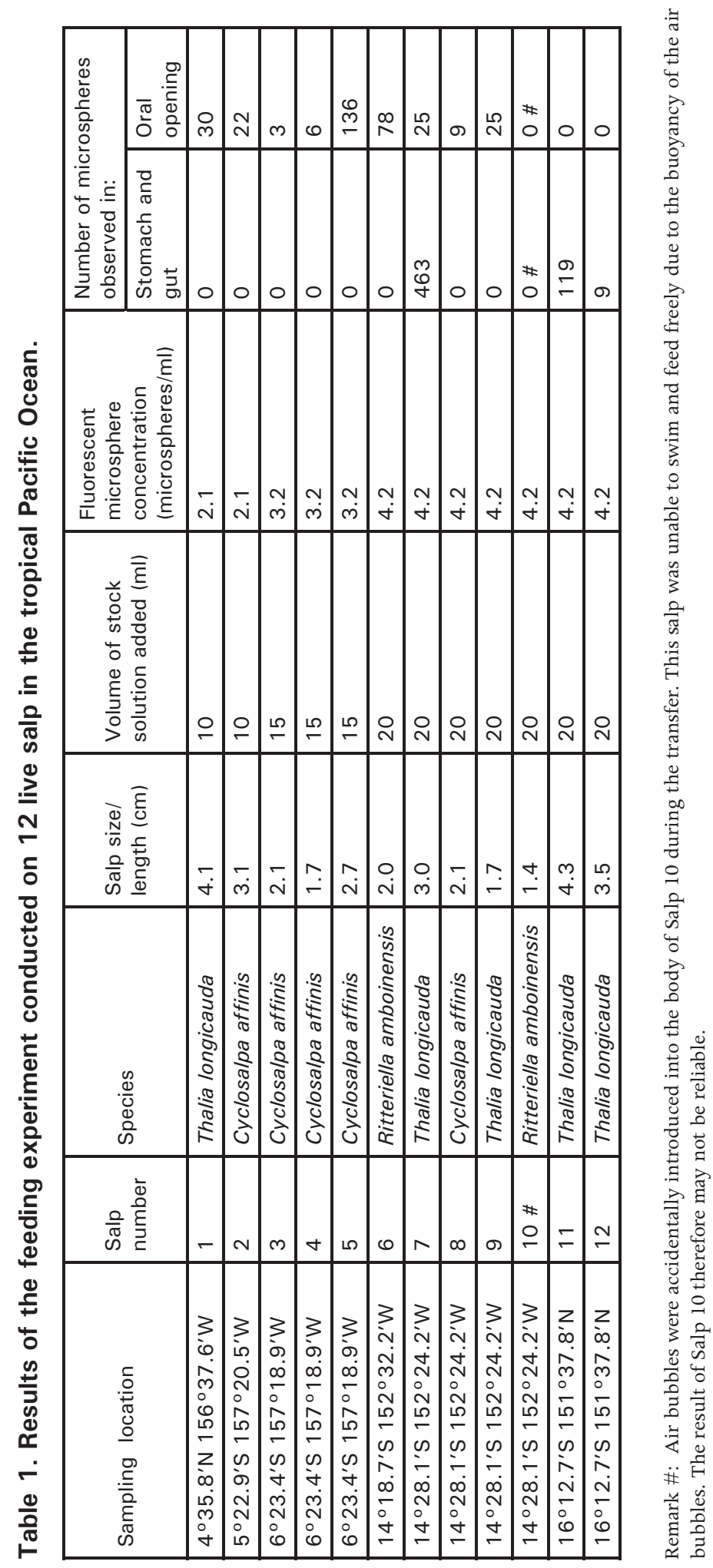




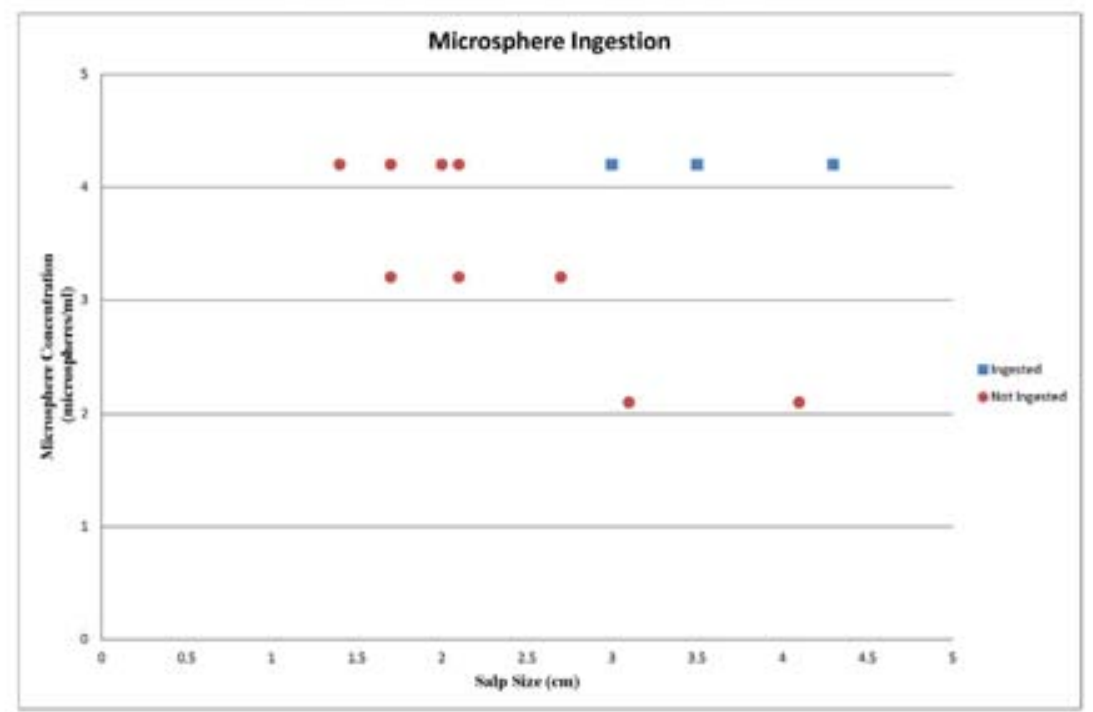

Figure 4.1. Relations between salp size, microsphere concentration and microsphere ingestion.

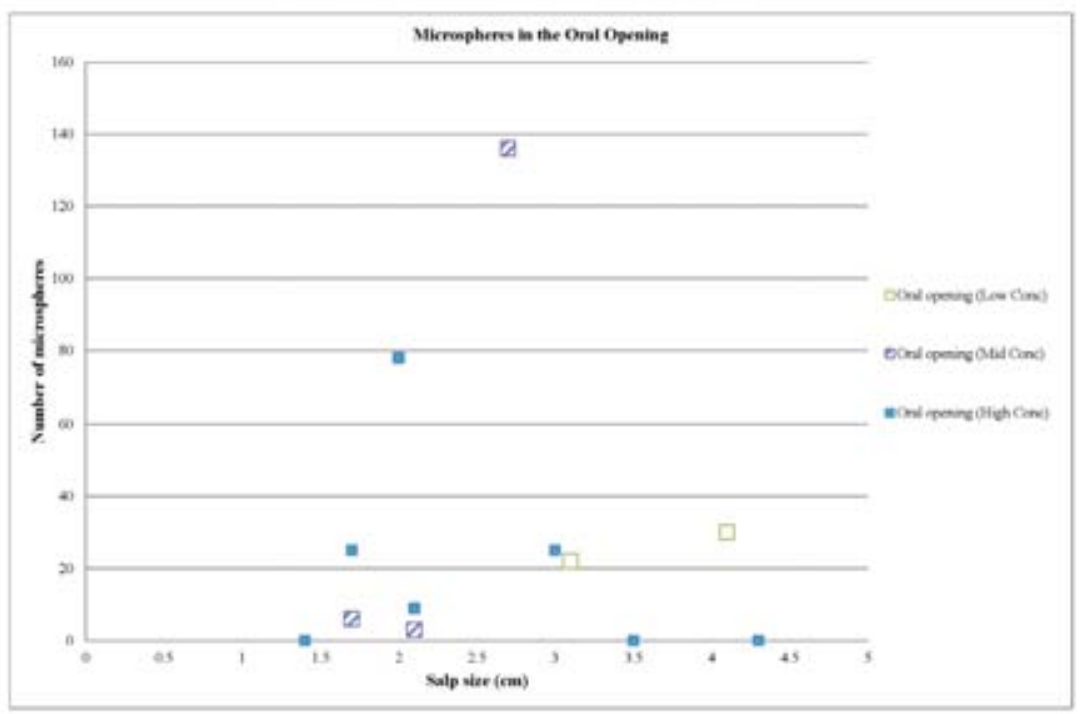

Figure 4.2. Relations between salp size, microsphere concentration and cluster of microspheres in the oral opening. 

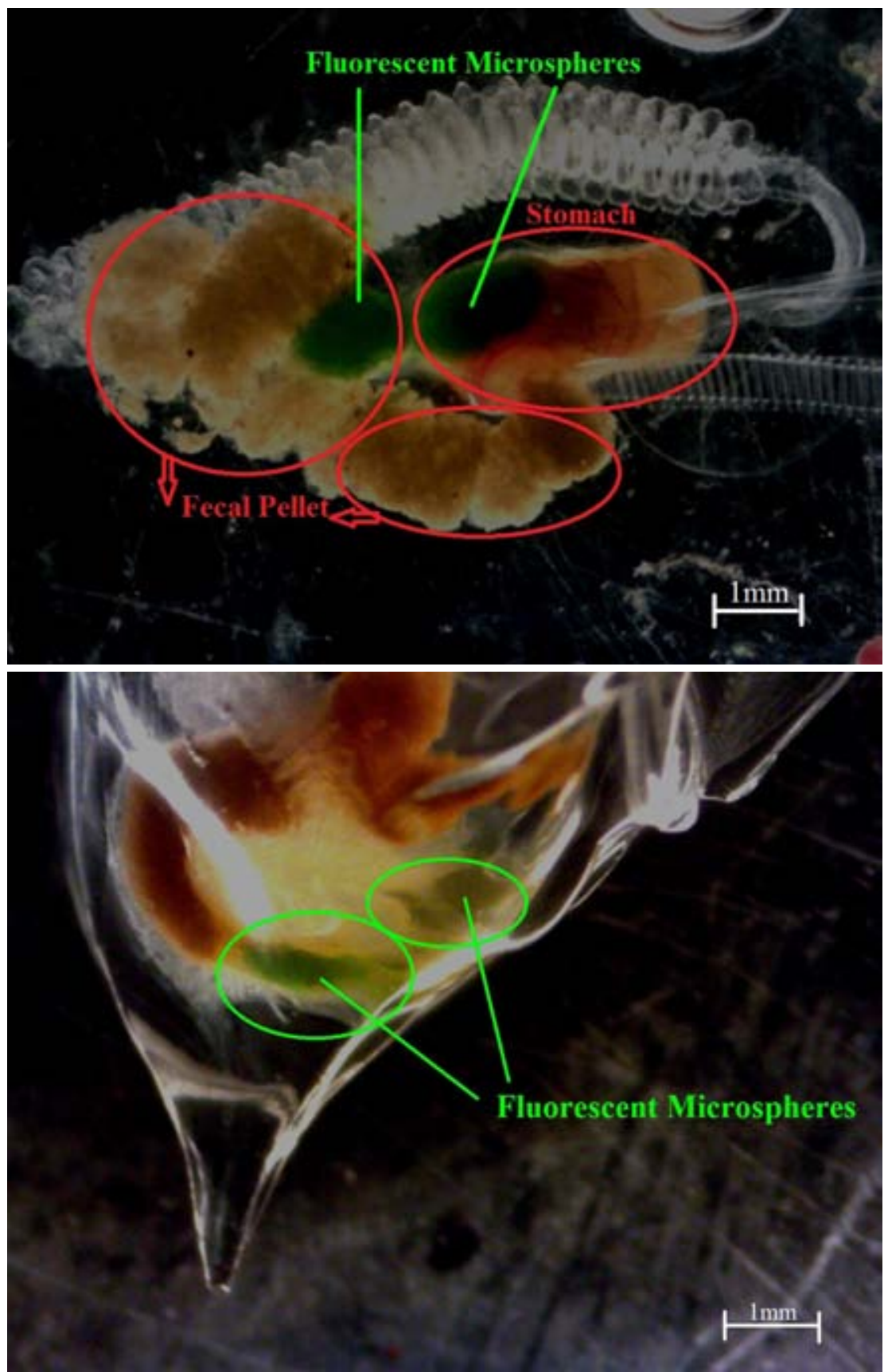

Figures 5.1 and 5.2. Ingestion of fluorescent microspheres by Salp 7 ( $3 \mathrm{~cm}$, top) and Salp $11(4.3 \mathrm{~cm}$, bottom). Note the presence of fluorescent microspheres in their stomach and the package of microspheres in the fecal pellet of Salp 7. 


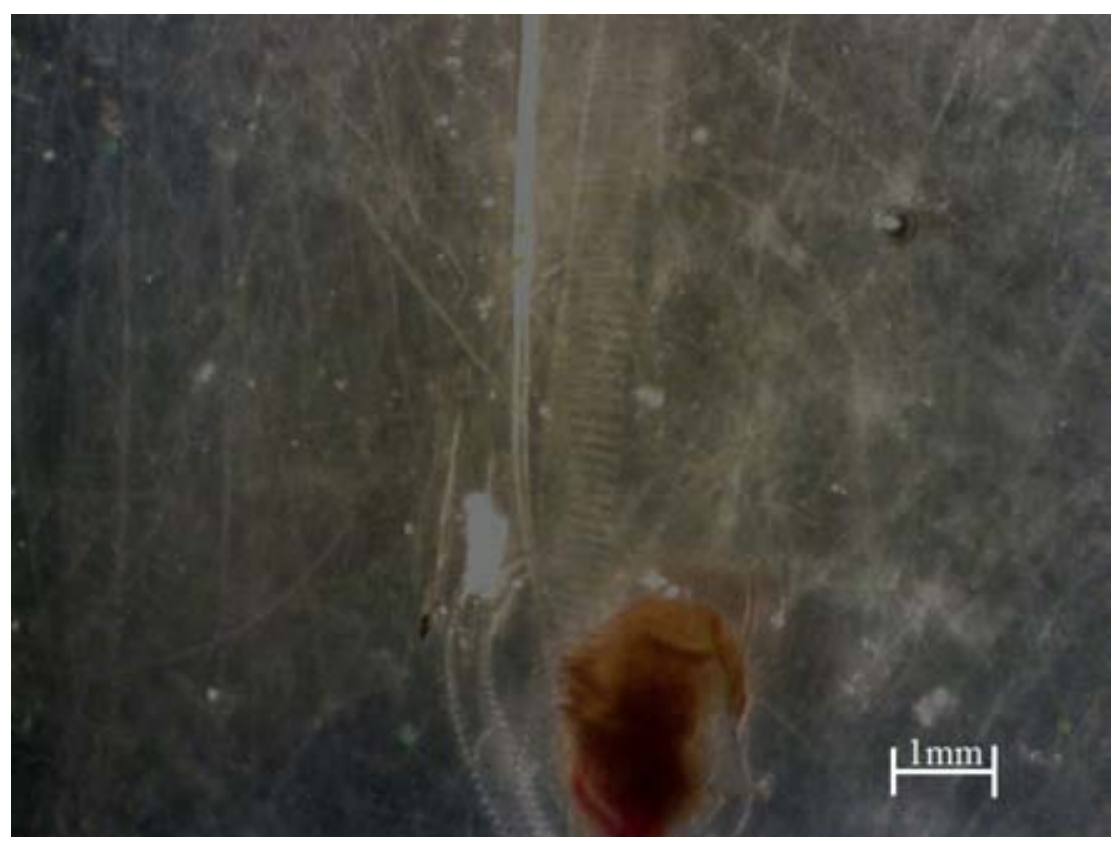

Figure 6. Absence of microspheres ingestion in smaller-sized Salp 8 (2.1 $\mathrm{cm})$. Note the absence of fluorescent microspheres in its gut or stomach

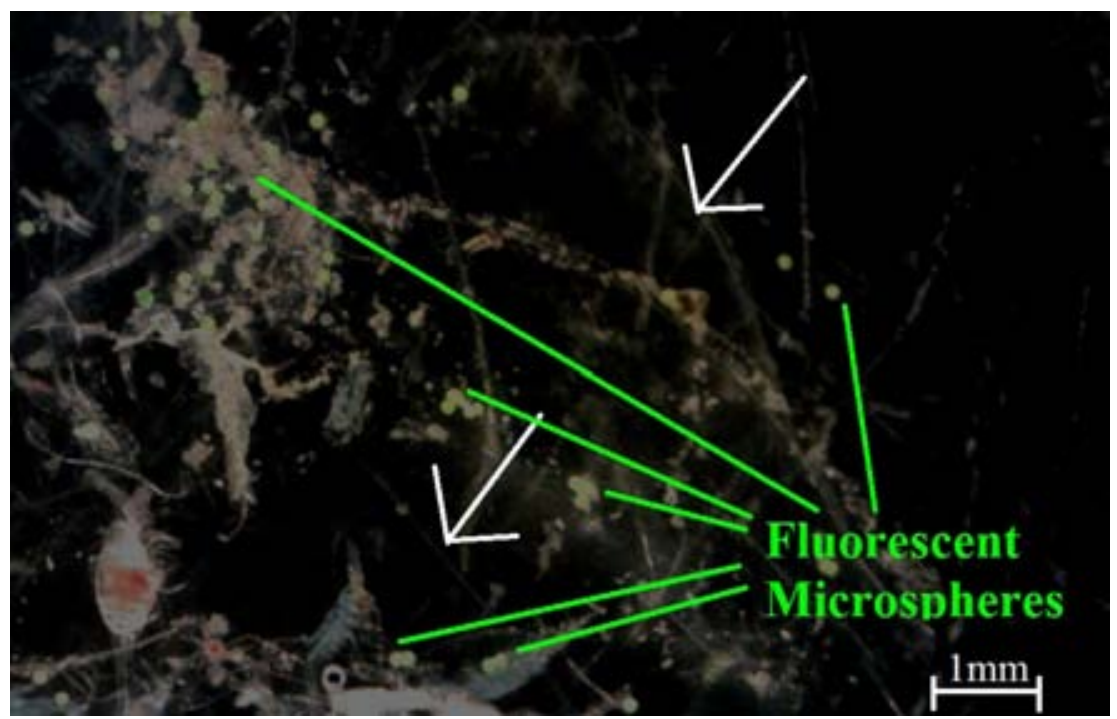

Figure 7. Clusters of fluorescent microspheres in the oral opening of smaller-sized Salp $6(2 \mathrm{~cm})$. The white arrows indicate the direction of water passing through the oral opening. 


\section{Discussion}

\subsection{Salp size, microsphere size, microsphere concentration and ingestion}

The results of the study suggest a size-specific response of salp to the presence of fluorescent microspheres. Ingestion of microspheres occurred exclusively in larger-sized salp (i.e. $\geq 3 \mathrm{~cm}$ ) that were subjected to a high microsphere concentration. Although no ingestion of microspheres occurred in the smallersized salp (i.e. $<3 \mathrm{~cm}$ ), clusters of microspheres were found in their oral openings at all given microsphere concentrations (i.e. medium to high).

It is possible that ingestion would only occur when the size of the microsphere is similar to that of the salp's normal prey. Normal prey of salp that were 0.4$1.37 \mathrm{~cm}$ in body size has been reported to be between 5-62 $\mu \mathrm{m}$ (Vargas \& Madin, 2004). Given the average microsphere size used in this study was $98.27 \mu \mathrm{m}$, these microspheres were likely to be closer to the size of normal prey of the largersized salp than the smaller-sized salp of the study. The clusters of microspheres observed in the oral openings of all the smaller-sized salp were likely to be a sign of clogging of their feeding apparatus due to exposure to microspheres larger than their normal prey. This observation is consistent with previous studies that reported small salp are unable to efficiently ingest large diatoms and ciliates, and these large prey can potentially clog up a small salp's feeding apparatus (Vargas \& Madin, 2004).

\subsection{Possible negative impact of microplastics on salp}

Based on the size-specific response of salp to the microspheres showed in this study, microplastics are likely to negatively affect salp of different sizes in different ways. For larger-sized salp, ingestion of microplastics is likely to occur at a high microplastic density. Salp that are $\sim 3-4.3 \mathrm{~cm}$ (i.e. the size of the largersized salp in this study) are expected to ingest microplastics of up to 90-106 $\mu \mathrm{m}$ in size (i.e. the size of the microspheres). The exact biological and physiological consequence of microplastic ingestion to filter-feeders is unknown. However, a reduction of nutrient gain and increase in body toxicity level can be expected. These negative impacts may lead to poor health and fecundity of salp, affecting their role in the carbon cycle. Furthermore, ingested microplastics are likely to be packaged into a salp's fecal pellet and subsequently sink into the deep ocean. This is supported by the package of microspheres into the fecal pellet of Salp 7. It is uncertain how the ocean and marine biota will be affected by this new phenomenon of downward microplastic flux, however, the introduction of microplastics to the benthic communities is likely to be negative. 
On the other hand, ingestion of microplastics is unlikely to occur in smallersized salp regardless of the microplastic concentrations. Based on the clusters of microspheres observed in the oral openings of the smaller-sized salp in this study, the major threat of microplastics to small salp is likely to be clogging of their feeding apparatus. Small salp that are $\sim 1.4-2.7 \mathrm{~cm}$ (i.e. the size of the smallersized salp in the study) are unlikely to ingest microplastics that are greater than 90-106 $\mu \mathrm{m}$ at any microplastic concentration. Since salp are unable to remove unwanted materials from their feeding apparatus (Harbison \& McAlister, 1979), its clogging is therefore likely to be detrimental to their survival, as well as to their capacity of continuous downward carbon flux in the carbon cycle.

The extent to which microplastics can negatively affect salp feeding depends on the microplastic concentration in the ocean. This concentration, however, is not constant throughout the ocean. In general, microplastic concentration inside a gyre is higher than that outside a gyre. For instance, a low microplastic concentration of 650 pieces $/ \mathrm{km}^{2}$ (pieces of plastic per square kilometer) was reported during the same cruise from Hawaii to Tahiti (Phalen \& Citron, 2011), yet a high microplastic concentration of 334,271 pieces $/ \mathrm{km}^{2}$ was reported in the North Pacific Subtropical Gyre (Howell et al., 2012). When comparing the above microplastic concentrations with that used in this study (i.e. 2.1-4.2 microspheres $/ \mathrm{ml}$ ), the current microplastic concentrations are relatively low and should have a limited negative impact on salp feeding. However, macroplastics will disintegrate over time and the number of microplastics is expected to multiply in the future. For this reason, the negative impact of microplastics on filter-feeders like salp is likely to intensify in the future and the problem must not be ignored.

\subsection{Limitations}

One potential bias in this study was the health conditions of salp in the feeding experiments. The majority of the larger-sized salp was in a better condition than smaller-sized salp and the former remained healthy throughout the feeding experiment. Some smaller-sized salp were less healthy and may have swum and fed less actively than their larger-sized counterparts. Nonetheless, since healthy and less healthy salp were present in both the larger-sized and smaller-sized salp category, the potential bias that was introduced due to differential health conditions is likely to be limited.

\section{Conclusion and recommendations}

In summary, the type of the negative impact of marine microplastics on filter-feeders like salp is expected to be size-dependent. While microplastic 
ingestion is likely to occur in larger-sized salp subjected to a high microplastic concentration, clogging of feeding apparatus is likely to occur in small-sized salp at medium to high microplastic concentrations. In both cases, the presence of pelagic microplastics is likely to have negative effects on their survival, feeding efficiency, fecundity, and their role in the carbon cycle. More studies are needed to examine the biological and physiological consequences of microplastics ingestion by filter-feeders like salp, as well as how these negative impacts may be magnified further up the food chain. Future studies are recommended using only healthy, active swimming salp for similar feeding experiments. Instead of a net tow, healthy, undamaged salp can be obtained by a SCUBA diver using a hand-held jar. In this way, a wider range of salp including less robust salp species and fragile smaller-sized salp can also be included in the study.

\section{Acknowledgements}

I would like to thank A. Scofield, C. Dykeman, M. Schrimpf for their help in collecting the samples and reviewing the manuscript, and to S. Bernasconi, $\mathrm{H}$. Walchak for their help in salp species identification. I acknowledge the skillful help of the captain, crews and all members of the S-238 SSV Robert C. Seamans. I also gratefully acknowledge the financial assistance received through the Sea Education Association's Eleanor and Fremont Chandler Presidential Scholarship and SEA Scholarship, as well as The Australian National University Vice Chancellor's Travel Grants.

\section{References}

Doyle, M. J., Watson, W., Bowlin, N. M., \& Sheavly, S. B. (2011). Plastic particles in coastal pelagic ecosystems of the Northeast Pacific ocean. Marine Environmental Research, 71(1), 41-52. doi:10.1016/j.marenvres.2010.10.001

Fortier, L., Le Fèvre, J., \& Legendre, L. (1994). Export of biogenic carbon to fish and to the deep ocean: the role of large planktonic microphages. Journal of Plankton Research, 16(7), 809 -839. doi:10.1093/plankt/16.7.809

Harbison, G. R., \& Gilmer, R. W. (1976). The Feeding Rates of the Pelagic Tunicate Pegea confederata and Two Other Salp. Limnology and Oceanography, 21(4), 517-528.

Harbison, G. R., \& McAlister, V. L. (1979). The Filter-Feeding Rates and Particle Retention Efficiencies of Three Species of Cyclosalpa (Tunicata, Thaliacea). Limnology and Oceanography, 24(5), 875-892. 
Howell, E. A., Bograd, S. J., Morishige, C., Seki, M. P., \& Polovina, J. J. (2012). On North Pacific circulation and associated marine debris concentration. Marine Pollution Bulletin, 65(1-3), 16-22. doi:10.1016/j.marpolbul.2011.04.034

Kaiser, J. (2010). The Dirt on Ocean Garbage Patches. Science, 328(5985), 1506. doi:10.1126/science.328.5985.1506

Moore, C. J., Moore, S. L., Leecaster, M. K., \& Weisberg, S. B. (2001). A comparison of plastic and plankton in the north Pacific central gyre. Marine Pollution Bulletin, 42(12), 1297-1300.

Nishikawa, J., \& Tsuda, A. (2001). Diel vertical migration of the tunicate Salpa thompsoni in the Southern Ocean during summer. Polar Biology, 24(4), 299302. doi:10.1007/s003000100227

Perissinotto, R., \& Pakhomov, E. A. (1998). Contribution of salp to carbon flux of marginal ice zone of the Lazarev Sea, Southern Ocean. Marine Biology, 131(1), 25-32. doi:10.1007/s002270050292

Phalen, W. G., \& Citron, E. H., (2011). Charting the Distribution of Plastic Debris in the Equatorial Central Pacific. Sea Education Association, Cruise S-238.

Ramaswamy, V., Sarin, M. M., \& Rengarajan, R. (2005). Enhanced export of carbon by salp during the northeast monsoon period in the northern Arabian Sea. Deep Sea Research Part II: Topical Studies in Oceanography, 52(14-15), 1922-1929. doi:doi: 10.1016/j.dsr2.2005.05.005

Sutherland, K. R., Madin, L. P., \& Stocker, R. (2010). Filtration of submicrometer particles by pelagic tunicates. Proceedings of the National Academy of Sciences, 107(34), 15129-15134. doi:10.1073/pnas.1003599107

Thompson, R. C., Olsen, Y., Mitchell, R. P., Davis, A., Rowland, S. J., John, A. W. G., McGonigle, D., et al. (2004). Lost at Sea: Where Is All the Plastic? Science, 304(5672), 838-838. doi:10.1126/science.1094559

Vargas, C. A., \& Madin, L. P. (2004). Zooplankton feeding ecology: clearance and ingestion rates of the salp Thalia democratica, Cyclosalpa affinis and Salpa cylindrica on naturally occurring particles in the Mid-Atlantic Bight. Journal of Plankton Research, 26(7), 827 -833. doi:10.1093/plankt/fbh068 